\title{
Research Article \\ On Exponential Stability Conditions of Descriptor Systems with Time-Varying Delay
}

\author{
S. Cong and Z.-B. Sheng \\ School of Mechanical E Electrical Engineering, Heilongjiang University, Harbin 150080, China \\ Correspondence should be addressed to S. Cong, shen_tsong@163.com
}

Received 8 August 2011; Revised 4 November 2011; Accepted 4 November 2011

Academic Editor: Kai Diethelm

Copyright (C) 2012 S. Cong and Z.-B. Sheng. This is an open access article distributed under the Creative Commons Attribution License, which permits unrestricted use, distribution, and reproduction in any medium, provided the original work is properly cited.

We are interested in the exponential stability of the descriptor system, which is composed of the slow and fast subsystems with time-varying delay. In computing a kind of Lyapunov functional, we employ a necessary number of slack matrices to render the balance and to yield the convexity condition for reducing the conservatism and dealing with the case of time-varying delay. Therefore, we can get the decay rate of the slow variables. Moreover, we characterize the effect of the fast subsystem on the derived decay rate and then prove the fast variables to decay exponentially through a perturbation approach. Finally, we provide an example to demonstrate the effectiveness of the method.

\section{Introduction}

Descriptor systems are also referred to as singular systems, generalized systems, differentialalgebraic systems, and so on. This kind of systems turns out to be precise to describe some practical systems that may undergo some extremal conditions, such as lossless transmission lines. Therefore, it has received considerable attentions to characterize the dynamics of such systems and develop the fundamental control theory in parallel with that of regular ones. In this respect, it has been proven to be a useful approach to decompose a descriptor system into slow and fast subsystems; see [1] and the references therein. Moreover, the existence of this kind of decomposition can be implied by some Lyapunov equality or Lyapunov inequality. Therefore, the Lyapunov method provides an efficient tool for both stability and stabilization problems; see, for example, [2-4].

Meanwhile, there have been great efforts dedicated to the study of time-delay systems since hysteresis is regarded as the important element in modeling many natural and artificial systems and it can be the source of instability and poor performance; see [5] and the references therein. In particular, in the presence of time-delay, the dynamics of a descriptor system can become rather complex; for example, the decomposition according to slow and 
fast subsystems no longer guarantees the absence of impulsive behavior; see [6]. For the stability problem of delayed descriptor systems, some sophisticated matrix transformation techniques have been posed and even extended to robust control problems so as to apply Lyapunov functional method and the associated convex optimization method under the constraint condition of reduced rank of matrix; see, for example, [6-13].

In this paper, we consider a class of descriptor systems with time-varying delay. The starting point is that the system under consideration satisfies some mild conditions so that it can be converted into the following differential-algebraic equations (see, e.g., $[8,11]$ ):

$$
\begin{gathered}
\dot{x}_{1}(t)=A x_{1}(t)+B_{11} x_{1}(t-h(t))+B_{12} x_{2}(t-h(t)), \\
0=x_{2}(t)+B_{21} x_{1}(x-h(t))+B_{22} x_{2}(t-h(t)) .
\end{gathered}
$$

This model is composed of the slow subsystem in (1.1) and the fast subsystem in (1.2). We refer to $x_{1}(t) \in R^{r}$ and $x_{2}(t) \in R^{n-r}$ as the slow and fast variables, respectively. In addition, $h(t) \in[0, h]$ is the delay with bounded varying rate $|\dot{h}(t)| \leq \mu<1$. The matrices $A, B_{11}, B_{12}, B_{21}$, and $B_{22}$ are of appropriate dimensions. To simplify typography, let us rewrite the system in (1.1) and (1.2) into the form of

$$
E \dot{x}(t)=\bar{A} x(t)+\bar{B} x(t-h(t))
$$

where $x(t)=\left[\begin{array}{l}x_{1}(t) \\ x_{2}(t)\end{array}\right], E=\operatorname{diag}\left\{I_{r}, 0\right\}, \bar{A}=\operatorname{diag}\left\{A, I_{n-r}\right\}$, and $\bar{B}=\left[\begin{array}{ll}B_{11} & B_{12} \\ B_{21} & B_{22}\end{array}\right]$. The goal of this paper is to establish conditions guaranteeing the zero solution of such a system to be exponentially stable.

Definition 1.1. System (1.3) is said to be exponentially stable if there exists an $\epsilon>0$ such that $\limsup _{t \rightarrow \infty} \ln \left|x\left(t ; x_{0}\right)\right| / t \leq-\epsilon$.

The most of the existing results on the stability problem of descriptor systems with delay only pertain to the case of constant delay. In short, as pointed out in [9], this is due to that time-varying delay makes it become hardly possible to explicitly express the fast variables. In [9], to tackle this problem, some terminologies have been borrowed from graph theory to model the dependency of the fast variables on past instants and express them in terms of the slow variables. This approach, however, is rather complicated for application and further improvement.

In this paper, we will focus on the case of time-varying and address the stability problem in such a way that we first get the decay rate of the slow variables by using Lyapunov functional approach and prove the stability of the fast subsystem through a perturbation approach. More precisely, we drop out the idea of expressing the fast variables but use them to perturb the derived decay rate and, therefore, get the conditions guaranteeing their convergence. To this end, we present a necessary number of slack matrices to produce some balance and convexity conditions, which can play a key role for reducing the conservatism caused by delay itself. 


\section{Main Results}

In what follows we need the following fact; see [14].

Lemma 2.1. The following statements are equivalent: (i) there is a positive-definite matrix $P$ such that $A^{\prime} P A<\gamma P, \gamma>0$; (ii) there are a symmetric matrix $P$ and a matrix $G$ such that $\left[\begin{array}{cc}\gamma P & A^{\prime} G^{\prime} \\ \star & G+G^{\prime}-P\end{array}\right]>0$.

To study the stability of system (1.1), we construct the Lyapunov functional as follows:

$$
V\left(x_{t}\right)=\sum_{k=1}^{6} V_{k}\left(x_{t}\right)
$$

with the terms defined as

$$
\begin{aligned}
& V_{1}\left(x_{t}\right)=x^{\prime}(t) E^{\prime} P \operatorname{Ex}(t), \\
& V_{2}\left(x_{t}\right)=2 x^{\prime}(t) E^{\prime} Q \int_{t-h}^{t} e^{\alpha(\theta-t)} x(\theta) \mathrm{d} \theta, \\
& V_{3}\left(x_{t}\right)=\int_{t-h}^{t} e^{\alpha(\theta-t)} x^{\prime}(\theta) \mathrm{d} \theta R \int_{t-h}^{t} e^{\alpha(\theta-t)} x(\theta) \mathrm{d} \theta, \\
& V_{4}\left(x_{t}\right)=\int_{t-h}^{t} e^{\alpha(\theta-t)} x^{\prime}(\theta) T x(\theta) \mathrm{d} \theta, \\
& V_{5}\left(x_{t}\right)=\int_{t-h(t)}^{t} e^{\alpha(\theta-t)} x^{\prime}(\theta) S x(\theta) \mathrm{d} \theta, \\
& V_{6}\left(x_{t}\right)=\int_{-h}^{0} \mathrm{~d} \theta \int_{t+\theta}^{t} e^{\alpha(\sigma-t)}\left[\begin{array}{c}
x(\sigma) \\
E \dot{x}(\sigma)
\end{array}\right]^{\prime}\left[\begin{array}{cc}
X & Y \\
\star & Z
\end{array}\right]\left[\begin{array}{c}
x(\sigma) \\
E \dot{x}(\sigma)
\end{array}\right] \mathrm{d} \sigma .
\end{aligned}
$$

Therefore, we can have

$$
\begin{aligned}
\dot{V}_{1}\left(x_{t}\right)= & 2 \dot{x}^{\prime}(t) E^{\prime}\left(U F_{1}+P E\right) x(t), \\
\dot{V}_{2}\left(x_{t}\right)+\alpha V_{2}\left(x_{t}\right)= & 2 \dot{x}^{\prime}(t) E^{\prime}\left(U F_{2}+Q\right) \int_{t-h}^{t} e^{\alpha(\theta-t)} x(\theta) \mathrm{d} \theta+2 x^{\prime}(t) E^{\prime} Q\left[x(t)-e^{-\alpha h} x(t-h)\right], \\
\dot{V}_{3}\left(x_{t}\right)+\alpha V_{3}\left(x_{t}\right)= & 2\left[x(t)-e^{-\alpha h} x(t-h)\right]^{\prime} R \int_{t-h}^{t} e^{\alpha(\theta-t)} x(\theta) \mathrm{d} \theta \\
& -\alpha \int_{t-h}^{t} e^{\alpha(\theta-t)} x^{\prime}(\theta) \mathrm{d} \theta R \int_{t-h}^{t} e^{\alpha(\theta-t)} x(\theta) \mathrm{d} \theta,
\end{aligned}
$$




$$
\begin{aligned}
\dot{V}_{4}\left(x_{t}\right)+\alpha V_{4}\left(x_{t}\right)= & x^{\prime}(t) T x(t)-e^{-\alpha h} x^{\prime}(t-h) T x(t-h), \\
\dot{V}_{5}\left(x_{t}\right)+\alpha V_{5}\left(x_{t}\right)= & x^{\prime}(t) S x(t)-(1-\dot{h}(t)) e^{-\alpha h(t)} x^{\prime}(t-h(t)) S x(t-h(t)), \\
\dot{V}_{6}\left(x_{t}\right)+\alpha V_{6}\left(x_{t}\right)= & h\left[\begin{array}{c}
x(t) \\
E \dot{x}(t)
\end{array}\right]^{\prime}\left[\begin{array}{cc}
X & Y \\
\star & Z
\end{array}\right]\left[\begin{array}{c}
x(t) \\
E \dot{x}(t)
\end{array}\right] \\
& -\int_{t-h}^{t} e^{\alpha(\theta-t)}\left[\begin{array}{c}
x(\theta) \\
E \dot{x}(\theta)
\end{array}\right]^{\prime}\left[\begin{array}{cc}
X & Y \\
\star & Z
\end{array}\right]\left[\begin{array}{c}
x(\theta) \\
E \dot{x}(\theta)
\end{array}\right] \mathrm{d} \theta,
\end{aligned}
$$

where $U$ is any square matrix satisfying $E^{\prime} U=0$ and $\operatorname{rank} U=n-r$, and, therefore, $F_{1}$ and $F_{2}$ can be any square matrices.

Theorem 2.2. Let $\gamma \in(0,1)$ and $\alpha=-\ln \gamma / h>0$. If there exist matrices $P, Q, R$ with $\left[\begin{array}{c}P \\ \star R \\ \star R\end{array}\right]>0$, $S>0, T>0, X, Y, Z$, and $L_{q}(q=1, \ldots, 6), H_{q}(q=1, \ldots, 4), G_{q}(q=1, \ldots, 6), F_{q}(q=1,2)$, and $M_{p q}, N_{p q}(p, q=1, \ldots, 4)$ such that the following matrix inequalities can be satisfied:

$$
\begin{gathered}
\Xi+(1-\gamma) \Gamma_{1} \leq 0, \\
\Xi+(1-\gamma) \Gamma_{2} \leq 0, \\
\Upsilon_{1}=\left[\begin{array}{cc}
\alpha \Omega_{1} & \Sigma_{1} \\
\star & \Pi
\end{array}\right] \geq 0, \\
\Upsilon_{2}=\left[\begin{array}{cc}
\alpha \Omega_{2} & \Sigma_{2} \\
\star & \Pi
\end{array}\right] \geq 0, \\
{\left[\begin{array}{cc}
\alpha\left(R+E L_{5}^{\prime}+E^{\prime} L_{5}\right) & L_{5}^{\prime}+\alpha E^{\prime} L_{6} \\
\star & L_{6}^{\prime}+L_{6}
\end{array}\right] \geq 0,}
\end{gathered}
$$

then the system in (1.3) is exponentially stable. Here, the matrices, the matrix blocks, and the matrix elements presented in (2.4)-(2.8) are defined as follows:

$$
\begin{aligned}
\Xi & =\left[\begin{array}{cccc}
\Xi_{11} & \Xi_{12} & \Xi_{13} & \Xi_{14} \\
\star & \Xi_{22} & \Xi_{23} & \Xi_{24} \\
\star & \star & \Xi_{33} & \Xi_{34} \\
\star & \star & \star & \Xi_{44}
\end{array}\right] ; \quad \Pi=\left[\begin{array}{cc}
X & Y \\
\star & Z
\end{array}\right] ; \\
\Gamma_{1} & =\left[\begin{array}{cccc}
M_{11} & M_{12} & M_{13}+L_{1}^{\prime} E & M_{14} \\
\star & M_{22} & M_{23}+L_{2}^{\prime} E & M_{24} \\
\star & \star & M_{33}+L_{3}^{\prime} E+E^{\prime} L_{3}+(1-\mu) S & M_{34}+E^{\prime} L_{4} \\
\star & \star & \star & M_{44}
\end{array}\right] ;
\end{aligned}
$$


Journal of Applied Mathematics

$$
\begin{aligned}
& \Gamma_{2}=\left[\begin{array}{cccc}
N_{11} & N_{12} & N_{13}+H_{1}^{\prime} E & N_{14} \\
\star & N_{22} & N_{23}+H_{2}^{\prime} E & N_{24} \\
\star & \star & N_{33}+H_{3}^{\prime} E+E^{\prime} H_{3} & N_{34}+E^{\prime} H_{4} \\
\star & \star & \star & N_{44}
\end{array}\right] \\
& \Omega_{1}=\left[\begin{array}{cccc}
M_{11} & M_{12} & M_{13} & M_{14} \\
\star & M_{22} & M_{23} & M_{24} \\
\star & \star & M_{33} & M_{34} \\
\star & \star & \star & M_{44}
\end{array}\right] ; \quad \Omega_{2}=\left[\begin{array}{cccc}
N_{11} & N_{12} & N_{13} & N_{14} \\
\star & N_{22} & N_{23} & N_{24} \\
\star & \star & N_{33} & N_{34} \\
\star & \star & \star & N_{44}
\end{array}\right] ; \\
& \Sigma_{1}=-\left[\begin{array}{cc}
-\bar{A}^{\prime} G_{5}+R+E^{\prime} L_{5}-\alpha L_{1}^{\prime} E & E^{\prime} L_{6}-L_{1}^{\prime}-\bar{A}^{\prime} G_{6} \\
-\bar{B}^{\prime} G_{5}+\left(U F_{2}+Q\right)-\alpha L_{2}^{\prime} E & -\bar{B}^{\prime} G_{6}-L_{2}^{\prime} \\
-\alpha L_{3}^{\prime} E-(1-\gamma) E^{\prime} L_{5} & -L_{3}^{\prime}-(1-\gamma) E^{\prime} L_{6} \\
-\gamma\left(R+E^{\prime} L_{5}\right)-\alpha L_{4}^{\prime} E & -\gamma E^{\prime} L_{6}-L_{4}^{\prime}
\end{array}\right] ; \\
& \Sigma_{2}=-\left[\begin{array}{cc}
-\bar{A}^{\prime} G_{5}+R+E^{\prime} L_{5}-\alpha H_{1}^{\prime} E & E^{\prime} L_{6}-H_{1}^{\prime}-\bar{A}^{\prime} G_{6} \\
-\bar{B}^{\prime} G_{5}+\left(U F_{2}+Q\right)-\alpha H_{2}^{\prime} E & -\bar{B}^{\prime} G_{6}-H_{2}^{\prime} \\
-\alpha H_{3}^{\prime} E-(1-\gamma) E^{\prime} L_{5} & -H_{3}^{\prime}-(1-\gamma) E^{\prime} L_{6} \\
-\gamma\left(R+E^{\prime} L_{5}\right)-\alpha H_{4}^{\prime} E & -\gamma E^{\prime} L_{6}-H_{4}^{\prime}
\end{array}\right] \text {; } \\
& \Xi_{11}=-\bar{A}^{\prime} G_{1}-G_{1}^{\prime} \bar{A}+\alpha E^{\prime} P E+L_{1}^{\prime} E+E^{\prime} L_{1}+E Q^{\prime}+Q E^{\prime}+S+T+h X ; \\
& \Xi_{12}=E^{\prime} L_{2}+\left(U F_{1}+P E\right)^{\prime}+G_{1}^{\prime}-\bar{A}^{\prime} G_{2}+h Y \text {; } \\
& \Xi_{13}=\gamma H_{1}^{\prime} E+E^{\prime} L_{3}-E^{\prime} L_{1}-G_{1}^{\prime} \bar{B}-\bar{A}^{\prime} G_{3} \text {; } \\
& \Xi_{14}=E^{\prime} L_{4}-\gamma\left(H_{1}^{\prime} E+E^{\prime} Q\right)-\bar{A}^{\prime} G_{4} ; \\
& \Xi_{22}=G_{2}^{\prime}+G_{2}+h Z \text {; } \\
& \Xi_{23}=-L_{2}^{\prime} E+\gamma H_{2}^{\prime} E+G_{3}-G_{2}^{\prime} \bar{B} ; \\
& \Xi_{24}=-\gamma H_{2}^{\prime} E+G_{4} ; \\
& \Xi_{33}=\gamma\left(H_{3}^{\prime} E+E^{\prime} H_{3}\right)-L_{3}^{\prime} E-E^{\prime} L_{3}-(1-\mu) S-\overline{B^{\prime}} G_{3}-G_{3}^{\prime} \bar{B} ; \\
& \Xi_{34}=-E^{\prime} L_{4}+\gamma E^{\prime} H_{4}-\gamma H_{3}^{\prime} E-\bar{B}^{\prime} G_{4} ; \\
& \Xi_{44}=-\gamma\left(T+H_{4}^{\prime} E+E^{\prime} H_{4}\right) \text {. }
\end{aligned}
$$

Proof. The first thing we have to do is to note that $\sum_{k=1}^{5} V_{k}\left(x_{t}\right)$ is positive-definite, and the positiveness of $V_{6}\left(x_{t}\right)$ is guaranteed simultaneously by (2.6) and (2.7). The subsequent proof is rather long; so for the clarity we will proceed in two steps. The first one concentrates on 
getting the decay rate for the slow variables, while the second one turns the focus on proving the fast variables eventually fallen into decay.

Step 1 (Present the decay rate of the slow variables). Combining (2.3) gives

$$
\begin{aligned}
\dot{V}\left(x_{t}\right) & +\alpha V\left(x_{t}\right) \\
\leq & 2 \dot{x}^{\prime}(t) E^{\prime}\left(U F_{1}+P E\right) x(t)+2 \dot{x}^{\prime}(t) E^{\prime}\left(U F_{2}+Q\right) \int_{t-h}^{t} e^{\alpha(\theta-t)} x(\theta) \mathrm{d} \theta \\
& +2\left[x(t)-e^{-\alpha h} x(t-h)\right]^{\prime}\left[Q^{\prime} E x(t)+R \int_{t-h}^{t} e^{\alpha(\theta-t)} x(\theta) \mathrm{d} \theta\right] \\
& +x^{\prime}(t)(S+T) x(t)-e^{-\alpha h} x^{\prime}(t-h) T x(t-h) \\
& +(1-\mu)\left(1-e^{-\alpha h(t)}\right) x^{\prime}(t-h(t)) S x(t-h(t))-(1-\mu) x^{\prime}(t-h(t)) S x(t-h(t)) \\
& +h\left[\begin{array}{c}
x(t) \\
E \dot{x}(t)
\end{array}\right]^{\prime}\left[\begin{array}{cc}
X & Y \\
\star & Z
\end{array}\right]\left[\begin{array}{c}
x(t) \\
E \dot{x}(t)
\end{array}\right]-\int_{t-h}^{t} e^{\alpha(\theta-t)}\left[\begin{array}{c}
x(\theta) \\
E \dot{x}(\theta)
\end{array}\right]^{\prime}\left[\begin{array}{ll}
X & Y \\
\star & Z
\end{array}\right]\left[\begin{array}{c}
x(\theta) \\
E \dot{x}(\theta)
\end{array}\right] \mathrm{d} \theta \\
& +\alpha x^{\prime}(t) E^{\prime} P E x(t)-\alpha \int_{t-h}^{t} e^{\alpha(\theta-t)} x^{\prime}(\theta) \mathrm{d} \theta R \int_{t-h}^{t} e^{\alpha(\theta-t)} x(\theta) \mathrm{d} \theta .
\end{aligned}
$$

In addition, partitioning the interval $[-h, 0]$ into the union of $[-h,-h(t)]$ and $[-h(t), 0]$, by integrating by parts we therefore obtain that

$$
\begin{gathered}
E\left[x(t)-e^{-\alpha h(t)} x(t-h(t))-\int_{t-h(t)}^{t} \alpha e^{\alpha(\theta-t)} x(\theta) \mathrm{d} \theta-\int_{t-h(t)}^{t} e^{\alpha(\theta-t)} \dot{x}(\theta) \mathrm{d} \theta\right]=0, \\
E\left[e^{-\alpha h(t)} x(t-h(t))-e^{-\alpha h} x(t-h)-\int_{t-h}^{t-h(t)} \alpha e^{\alpha(\theta-t)} x(\theta) \mathrm{d} \theta-\int_{t-h}^{t-h(t)} e^{\alpha(\theta-t)} \dot{x}(\theta) \mathrm{d} \theta\right]=0 .
\end{gathered}
$$

It is known that inserting some slack matrices into computing the constructed Lyapunov functional can produce some balance and convexity conditions. Doing this, we combine (2.11) into the identity as follows:

$$
\begin{aligned}
& 0 \equiv 2\left[x(t)+\left(1-e^{-\alpha h(t)}\right) x(t-h(t))-x(t-h(t))-\int_{t-h(t)}^{t} \alpha e^{\alpha(\theta-t)} x(\theta) \mathrm{d} \theta\right. \\
& \left.\quad-\int_{t-h(t)}^{t} e^{\alpha(\theta-t)} \dot{x}(\theta) \mathrm{d} \theta\right]^{\prime} \\
& \times E^{\prime}\left[L_{1} x(t)+L_{2} E \dot{x}(t)+L_{3} x(t-h(t))+L_{4} x(t-h)\right. \\
& \left.\quad+L_{5} \int_{t-h}^{t} e^{\alpha(\theta-t)} x(\theta) \mathrm{d} \theta+L_{6} \int_{t-h}^{t} e^{\alpha(\theta-t)} E \dot{x}(\theta) \mathrm{d} \theta\right]
\end{aligned}
$$


Journal of Applied Mathematics

$$
\begin{aligned}
& +2\left[\left(e^{-\alpha h(t)}-e^{-\alpha h}\right) x(t-h(t))+e^{-\alpha h} x(t-h(t))-e^{-\alpha h} x(t-h)-\int_{t-h}^{t-h(t)} \alpha e^{\alpha(\theta-t)} x(\theta) \mathrm{d} \theta\right. \\
& \left.-\int_{t-h}^{t-h(t)} e^{\alpha(\theta-t)} \dot{x}(\theta) \mathrm{d} \theta\right]^{\prime} \\
& \times E^{\prime}\left[H_{1} x(t)+H_{2} E \dot{x}(t)+H_{3} x(t-h(t))+H_{4} x(t-h)\right. \\
& \left.+L_{5} \int_{t-h}^{t} e^{\alpha(\theta-t)} x(\theta) \mathrm{d} \theta+L_{6} \int_{t-h}^{t} e^{\alpha(\theta-t)} E \dot{x}(\theta) \mathrm{d} \theta\right] \\
& =2\left[x(t)-x(t-h(t))-\int_{t-h(t)}^{t} \alpha e^{\alpha(\theta-t)} x(\theta) \mathrm{d} \theta-\int_{t-h(t)}^{t} e^{\alpha(\theta-t)} \dot{x}(\theta) \mathrm{d} \theta\right]^{\prime} \\
& \times E^{\prime}\left[L_{1} x(t)+L_{2} E \dot{x}(t)+L_{3} x(t-h(t))+L_{4} x(t-h)\right] \\
& +2\left[e^{-\alpha h} x(t-h(t))-e^{-\alpha h} x(t-h)-\int_{t-h}^{t-h(t)} \alpha e^{\alpha(\theta-t)} x(\theta) \mathrm{d} \theta-\int_{t-h}^{t-h(t)} e^{\alpha(\theta-t)} \dot{x}(\theta) \mathrm{d} \theta\right]^{\prime} \\
& \times E^{\prime}\left[H_{1} x(t)+H_{2} E \dot{x}(t)+H_{3} x(t-h(t))+H_{4} x(t-h)\right] \\
& +2\left[x(t)-\left(1-e^{-\alpha h}\right) x(t-h(t))-e^{-\alpha h} x(t-h)\right]^{\prime} E^{\prime} \\
& \times\left[L_{5} \int_{t-h}^{t} e^{\alpha(\theta-t)} x(\theta) \mathrm{d} \theta+L_{6} \int_{t-h}^{t} e^{\alpha(\theta-t)} E \dot{x}(\theta) \mathrm{d} \theta\right] \\
& -2\left[E \int_{t-h}^{t} \alpha e^{\alpha(\theta-t)} x(\theta) \mathrm{d} \theta+\int_{t-h}^{t} e^{\alpha(\theta-t)} E \dot{x}(\theta) \mathrm{d} \theta\right]^{\prime} \\
& \times\left[L_{5} \int_{t-h}^{t} e^{\alpha(\theta-t)} x(\theta) \mathrm{d} \theta+L_{6} \int_{t-h}^{t} e^{\alpha(\theta-t)} E \dot{x}(\theta) \mathrm{d} \theta\right] \\
& +2\left(1-e^{-\alpha h(t)}\right) x^{\prime}(t-h(t)) E^{\prime}\left[L_{1} x(t)+L_{2} E \dot{x}(t)+L_{3} x(t-h(t))+L_{4} x(t-h)\right] \\
& +2\left(e^{-\alpha h(t)}-e^{-\alpha h}\right) x^{\prime}(t-h(t)) E^{\prime}\left[H_{1} x(t)+H_{2} E \dot{x}(t)+H_{3} x(t-h(t))+H_{4} x(t-h)\right] .
\end{aligned}
$$

Furthermore, we can have additional slack matrices through the following identity

$$
\begin{aligned}
0 \equiv 2[E \dot{x}(t)-\bar{A} x(t)-\bar{B} x(t-h(t))]^{\prime} \\
\times \\
\quad \times\left[G_{1} x(t)+G_{2} E \dot{x}(t)+G_{3} x(t-h(t))+G_{4} x(t-h)+G_{5} \int_{t-h}^{t} e^{\alpha(\theta-t)} x(\theta) \mathrm{d} \theta\right. \\
\left.\quad+G_{6} \int_{t-h}^{t} e^{\alpha(\theta-t)} E \dot{x}(\theta) \mathrm{d} \theta\right] .
\end{aligned}
$$


Therefore, substituting (2.12) and (2.13) into the right-hand side of (2.10) and rearranging the obtained terms according to the augmented system variables as

$$
\begin{gathered}
\eta(t)=\left[\begin{array}{llll}
x^{\prime}(t) & E \dot{x}(t) & x^{\prime}(t-h(t)) & x^{\prime}(t-h)
\end{array}\right]^{\prime}, \\
\zeta_{t}(\theta)=\left[\begin{array}{lll}
\eta^{\prime}(t) & x^{\prime}(t+\theta) & E^{\prime} \dot{x}^{\prime}(t+\theta)
\end{array}\right]^{\prime}
\end{gathered}
$$

yields

$$
\begin{aligned}
\dot{V}\left(x_{t}\right) & +\alpha V\left(x_{t}\right) \\
\leq & \eta^{\prime}(t) \Xi \eta(t)+2\left(1-e^{-\alpha h(t)}\right) x^{\prime}(t-h(t)) E^{\prime}\left[L_{1} x(t)+L_{2} E \dot{x}(t)+L_{3} x(t-h(t))+L_{4} x(t-h)\right] \\
& +2\left(e^{-\alpha h(t)}-e^{-\alpha h}\right) x^{\prime}(t-h(t)) E^{\prime}\left[H_{1} x(t)+H_{2} E \dot{x}(t)+H_{3} x(t-h(t))+H_{4} x(t-h)\right] \\
& -\int_{-h(t)}^{0} e^{\alpha \theta} \zeta_{t}^{\prime}(\theta)\left[\begin{array}{cc}
0 & \Sigma_{1} \\
\star & \Pi
\end{array}\right] \zeta_{t}(\theta) \mathrm{d} \theta-\int_{-h}^{-h(t)} e^{\alpha \theta} \zeta_{t}^{\prime}(\theta)\left[\begin{array}{cc}
0 & \Sigma_{2} \\
\star & \Pi
\end{array}\right] \zeta_{t}(\theta) \mathrm{d} \theta \\
& -\int_{t-h}^{t} e^{\alpha(\theta-t)}\left[\begin{array}{c}
x(\theta) \\
E \dot{x}(\theta)
\end{array}\right]^{\prime} \mathrm{d} \theta\left[\begin{array}{cc}
\alpha\left(R+E L_{5}^{\prime}+E^{\prime} L_{5}\right) & L_{5}^{\prime}+\alpha E^{\prime} L_{6} \\
\star & L_{6}^{\prime}+L_{6}
\end{array}\right] \int_{t-h}^{t} e^{\alpha(\theta-t)}\left[\begin{array}{c}
x(\theta) \\
E \dot{x}(\theta)
\end{array}\right] \mathrm{d} \theta \\
& +\left[\left(1-e^{-\alpha h(t)}\right)-\alpha \int_{t-h(t)}^{t} e^{\alpha(\theta-t)} \mathrm{d} \theta\right] \eta^{\prime}(t) \Omega_{1} \eta(t) \\
& +\left[\left(e^{-\alpha h(t)}-e^{-\alpha h}\right)-\alpha \int_{t-h}^{t-h(t)} e^{\alpha(\theta-t)} \mathrm{d} \theta\right] \eta^{\prime}(t) \Omega_{2} \eta(t) .
\end{aligned}
$$

By using (2.8) to drop the sixth term and arranging the remaining terms on the right-hand side, (2.15) becomes

$$
\begin{aligned}
\dot{V}\left(x_{t}\right)+\alpha V\left(x_{t}\right) \leq & \eta^{\prime}(t)\left[\Xi+\left(1-e^{-\alpha h(t)}\right) \Gamma_{1}+\left(e^{-\alpha h(t)}-e^{-\alpha h}\right) \Gamma_{2}\right] \eta(t) \\
& -\int_{-h(t)}^{0} e^{\alpha \theta} \zeta_{t}^{\prime}(\theta) \Upsilon_{1} \zeta_{t}(\theta) \mathrm{d} \theta-\int_{-h}^{-h(t)} e^{\alpha \theta} \zeta_{t}^{\prime}(\theta) \Upsilon_{2} \zeta_{t}(\theta) \mathrm{d} \theta
\end{aligned}
$$

Thus, recalling (2.6) and (2.7), we have

$$
\dot{V}\left(x_{t}\right)+\alpha V\left(x_{t}\right) \leq \eta^{\prime}(t)\left[\Xi+\left(1-e^{-\alpha h(t)}\right) \Gamma_{1}+\left(e^{-\alpha h(t)}-e^{-\alpha h}\right) \Gamma_{2}\right] \eta(t) .
$$

The right-hand side of (2.17) turns out to be convex in $e^{-\alpha h(t)}$. It then can be eliminated through the boundary conditions (2.4) and (2.5), which correspond to the cases of $e^{-\alpha h}=\gamma$ and $e^{-\alpha 0}=1$, respectively. We then obtain

$$
\dot{V}\left(x_{t}\right) \leq-\alpha V\left(x_{t}\right)
$$


And hence,

$$
x_{1}^{\prime}(t) P_{11} x_{1}(t) \leq V\left(x_{0}\right) e^{-\alpha t}, \quad t \geq 0,
$$

where $P_{11}$ is the first $r \times r$ order principle block of the matrix $P$. Therefore, it is proven that the slow subsystem is exponentially stable.

Step 2 (Prove the fast variables fallen into decay exponentially). We proceed in such a way that we first conclude the Schur stability of the difference equation $x_{2}(t)+B_{22} x_{2}(t-h(t))=0$, and, therefore, prove the fast variables eventually fallen into decay via evaluating their effect on the decay rate derived as in (2.19) From (2.4) or (2.5), it is straightforward to see

$$
\Phi=\left[\begin{array}{ccc}
\Phi_{11} & \Phi_{12} & \Phi_{13} \\
\star & \Phi_{22} & \Phi_{23} \\
\star & \star & \Phi_{33}
\end{array}\right] \leq 0,
$$

where $\Phi_{11}=-\bar{A}^{\prime} G_{1}-G_{1}^{\prime} \bar{A}+\alpha E^{\prime} P E+L_{1}^{\prime} E+E^{\prime} L_{1}+E Q^{\prime}+Q E^{\prime}+S, \Phi_{12}=E^{\prime} L_{2}+\left(U F_{1}+P E\right)^{\prime}+$ $G_{1}^{\prime}-\bar{A}^{\prime} G_{2}, \Phi_{13}=\gamma H_{1}^{\prime} E+E^{\prime} L_{3}-E^{\prime} L_{1}-G_{1}^{\prime} \bar{B}-\bar{A}^{\prime} G_{3}+(1-\gamma) L_{1}^{\prime} E, \Phi_{22}=G_{2}^{\prime}+G_{2}, \Phi_{23}=$ $-L_{2}^{\prime} E+\gamma H_{2}^{\prime} E+G_{3}-G_{2}^{\prime} \bar{B}+(1-\gamma) L_{2}^{\prime} E$, and $\Phi_{33}=\gamma\left(H_{3}^{\prime} E+E^{\prime} H_{3}-L_{3}^{\prime} E-E^{\prime} L_{3}-(1-\mu) S\right)-\bar{B}^{\prime} G_{3}-G_{3}^{\prime} \bar{B}$.

Pre- and postmultiplying (2.20) by the matrix $\left[\begin{array}{ll}I & \vec{A}^{\prime} \\ 0 \vec{B}^{\prime} & I\end{array}\right]$ and its transpose, respectively,
that we get that

$$
\Psi=\left[\begin{array}{cc}
\Psi_{11} & \Psi_{12} \\
\star & \Psi_{22}
\end{array}\right] \leq 0,
$$

where $\Psi_{11}=\alpha E^{\prime} P E+L_{1}^{\prime} E+E^{\prime} L_{1}+E Q^{\prime}+Q E^{\prime}+S+\bar{A}^{\prime} L_{2}^{\prime} E+E^{\prime} L_{2} \bar{A}+\bar{A}^{\prime} P E+E^{\prime} P \bar{A}+\bar{A}^{\prime} U F_{1}+F_{1}^{\prime} U^{\prime} \bar{A}$, $\Psi_{12}=E^{\prime} L_{2}+E^{\prime} P \bar{B}+F_{1}^{\prime} U^{\prime} \bar{B}+\gamma H_{1}^{\prime} E+E^{\prime} L_{3}-E^{\prime} L_{1}+(1-\gamma) L_{1}^{\prime} E-\bar{A}^{\prime} L_{2} E+\gamma \bar{A}^{\prime} H_{2}^{\prime} E+(1-\gamma) \bar{A}^{\prime} L_{2}^{\prime} E$, and $\Psi_{22}=\gamma\left(H_{3}^{\prime} E+E^{\prime} H_{3}-L_{3}^{\prime} E-E^{\prime} L_{3}+\bar{B}^{\prime} H_{2}^{\prime} E+E^{\prime} H_{2} \bar{B}-\bar{B}^{\prime} L_{2}^{\prime} E-E^{\prime} L_{2} \bar{B}-(1-\mu) S\right)$.

In fact, the family of slack matrices $G_{q}(q=1, \ldots, 6)$ plays a key role in getting (2.21). Furthermore, noting the special structures of the matrices $E, U$, and $\bar{A}$, from (2.21) we can deduce that

$$
\left[\begin{array}{cc}
S_{22}+F_{1,22}^{\prime}+F_{1,22} & F_{1,22}^{\prime} B_{22} \\
\star & -\gamma(1-\mu) S_{22}
\end{array}\right] \leq 0
$$

where $S_{22}$ and $F_{1,22}$ represent the last $(n-r) \times(n-r)$-order principle blocks of of the matrices $S$ and $F_{1}$, respectively. According to Lemma 2.1, we have

$$
B_{22}^{\prime} S_{22} B_{22} \leq \gamma(1-\mu) S_{22} \leq \gamma S_{22}
$$

This constraint condition does imply that if the varying rate of delay exceeds 1 , the system in (1.1) would no more be able to retain the stability. 
By the fact that $\left|\xi_{1}+\xi_{2}\right|^{2} \leq\left|\xi_{1}\right|^{2} / \delta+\left|\xi_{2}\right|^{2} /(1-\delta)$ holds for all vectors $\xi_{1}, \xi_{2}$ of same dimension and number $\delta \in(0,1)$, from (2.19) and (2.23) we can estimate that

$$
\begin{aligned}
x_{2}^{\prime}(t) S_{22} x_{2}(t) & \leq \frac{1}{1-\delta} x_{1}^{\prime}(t-h(t)) B_{21}^{\prime} S_{22} B_{21} x_{1}(t-h(t))+\frac{1}{\delta} x_{2}^{\prime}(t-h(t)) B_{22}^{\prime} S_{22} B_{22} x_{2}(t-h(t)) \\
& \leq \frac{x^{V}\left(x_{0}\right)}{1-\delta} e^{-\alpha(t-h(t))}+\frac{r}{\delta} \sup _{t-h \leq \theta \leq t} x_{2}^{\prime}(t+\theta) S_{22} x_{2}(t+\theta) \\
& \leq \frac{x^{V}\left(x_{0}\right)}{r(1-\delta)} e^{-\alpha t}+\frac{\gamma}{\delta} \sup _{t-h \leq \theta \leq t} x_{2}^{\prime}(t+\theta) S_{22} x_{2}(t+\theta), \quad t \geq 0,
\end{aligned}
$$

where $\chi$ is sufficiently large so that $B_{21}^{\prime} S_{22} B_{21} \leq x P_{11}$ and $\delta$ is specified within $(\gamma, 1)$. Let $\psi_{k}=\sup _{(k-1) h \leq \theta \leq k h}\left\{x_{2}^{\prime}(t+\theta) S_{22} x_{2}(t+\theta)\right\}, k=0,1,2, \ldots$. Then, from (2.24) it follows that

$$
\psi_{k} \leq \frac{x V\left(x_{0}\right)}{\gamma(1-\delta)} e^{-\alpha(k-1) h}+\frac{\gamma}{\delta}\left(\psi_{k-1} \vee \psi_{k}\right), \quad k \geq 1
$$

Therefore,

$$
e^{\epsilon k h} \psi_{k} \leq \frac{x^{V}\left(x_{0}\right)}{\gamma(1-\delta)} e^{\alpha h-(\alpha-\epsilon) k h}+\frac{\gamma}{\delta} e^{\epsilon h}\left(e^{\epsilon(k-1) h} \psi_{k-1} \vee e^{\epsilon k h} \psi_{k}\right), \quad k \geq 1,
$$

where the positive number $\epsilon$ is sufficiently small for $\gamma e^{\epsilon h}<\delta$.

Noting $\epsilon<(\ln \delta-\ln \gamma) / h<\alpha$, we deduce that

$$
\max _{1 \leq i \leq k}\left(e^{\epsilon i h} \psi_{i}\right) \leq \frac{x^{V}\left(x_{0}\right)}{\gamma(1-\delta)} e^{\alpha h}+\frac{\gamma}{\delta} e^{e h}\left[\psi_{0}+\max _{1 \leq i \leq k}\left(e^{\epsilon i h} \psi_{i}\right)\right] .
$$

Hence,

$$
\max _{1 \leq i \leq k}\left(e^{\epsilon i h} \psi_{i}\right) \leq \sigma:=\frac{\left(\chi^{V}\left(x_{0}\right) /(1-\delta)+(\gamma / \delta) e^{\epsilon h} \psi_{0}\right)}{\left(1-(\gamma / \delta) e^{e h}\right)}
$$

Thus,

$$
\psi_{k} \leq \sigma e^{-\epsilon k h}, \quad k \geq 1
$$

But this implies that

$$
\limsup _{k \rightarrow \infty} \frac{\ln \psi_{k}}{k h} \leq-\epsilon
$$


Table 1: Calculated upper bound of size of delay for various varying rates.

\begin{tabular}{lccc}
\hline$\mu$ & 0.0 & 0.5 & 0.9 \\
$h$ & 2.130 & 1.741 & 1.336 \\
\hline
\end{tabular}

Table 2: Calculated upper bound of size of delay for various specified $\gamma$.

\begin{tabular}{lccccc}
\hline$r$ & 0.6 & 0.7 & 0.8 & 0.9 & 0.99 \\
$h$ & 1.786 & 1.895 & 1.990 & 2.071 & 2.137 \\
\hline
\end{tabular}

And it follows that

$$
\limsup _{t \rightarrow \infty} \frac{\ln \left|x_{2}(t)\right|^{2}}{t} \leq-\epsilon
$$

This together with (2.19) then completes the proof.

In arranging the augmented system variables, we insert a necessary number of slack matrices to render some balance and flexibility. Also, with the aid of slack matrices, the interval $[-h, 0]$ is decomposed into the union of $[-h,-h(t)]$ and $[-h(t), 0]$ and, moreover, the terms factored by $e^{-\alpha h(t)}$ are reformulated into a form of convex combination. In this way, the Lyapunov functional in (2.1) is computed almost without loss of its generality.

In the derived stability conditions, there only is a parameter to be specified, namely, $\gamma$, which deserves a brief discussion. In fact, as shown in (2.23), it is introduced to guarantee the difference equation $x_{2}(t)+B_{22} x_{2}(t-h(t))=0$ to be Schur stable. Therefore, we use $\gamma$ to characterize the effect of the fast subsystem on the decay rate of the slow variables, $\alpha$. Indeed, this turns out to be a typical perturbation approach to prove stability. Besides, $\gamma$ is one of boundary conditions of the convex combination on the right-hand side of (2.17).

\section{An Example}

In this section, we use a numerical example to demonstrate the theoretical results.

Example 3.1. Consider a system in the form of (1.3) with the following parameters:

$$
E=\left[\begin{array}{lll}
1 & 0 & 0 \\
0 & 1 & 0 \\
0 & 0 & 0
\end{array}\right], \quad \bar{A}=\left[\begin{array}{ccc}
0.5 & 0 & 0 \\
0 & 0.3 & 0 \\
0 & 0 & 1
\end{array}\right], \quad \bar{B}=\left[\begin{array}{ccc}
-1 & 0 & 0.2 \\
-1 & -1 & -0.4 \\
1 & 0 & 0.3
\end{array}\right]
$$

Specified $\gamma=0.98$, the calculated stability margins for various varying-rate of delay are presented in Table 1. On the other hand, we compute the upper bound of size of delay for various specified $\gamma$ with fixing $\mu=0$, which is shown in Table 2 . In the light of the discussion on the parameter $\gamma$, it would become clear that there is a mutually constraint relation between the difference operator $x_{2}(t)+B_{22} x_{2}(t-h(t))$ and the size of delay in guaranteeing stability. 


\section{Conclusion}

We considered a class of descriptor systems with time-varying delay. We developed a Lyapunov technique to investigate the exponential stability of such a system, which combines a necessary number of slack matrices, convexity condition, and matrix transformation. Therefore, after getting the decay rate for the slow variables, through a perturbation approach we came to the conclusion that the fast variables eventually fall into decay exponentially. A numerical example was given to illustrate the theoretical results.

\section{Acknowledgments}

The authors would like to thank the anonymous reviewers for the detailed and constructive comments that helped in improving this paper. This work is supported by the National Natural Science Foundation of China under Grant 60974027.

\section{References}

[1] L. Dai, Singular Control Systems, vol. 118 of Lecture Notes in Control and Information Sciences, Springer, Berlin, Germany, 1989.

[2] J. Y. Ishihara and M. H. Terra, "On the Lyapunov theorem for singular systems," IEEE Transactions on Automatic Control, vol. 47, no. 11, pp. 1926-1930, 2008.

[3] I. Masubuchi, Y. Kamitane, A. Ohara, and N. Suda, " $H_{\infty}$ control for descriptor systems: a matrix inequalities approach," Automatica, vol. 33, no. 4, pp. 669-673, 1997.

[4] E. Uezato and M. Ikeda, "Strict LMI conditions for stability, robust stabilization, and $H_{\infty}$ control of descriptor systems," in Proceedings of the 38th IEEE Conference on Decision and Control, pp. 4092-4097, Phoenix, Ariz, USA, 1999.

[5] K. Gu, V. L. Kharitonov, and J. Chen, Stability of Time-Delay Systems, Birkhäuser, Boston, Mass, USA, 2003.

[6] S. Zhu, C. Zhang, Z. Cheng, and J. Feng, "Delay-dependent robust stability criteria for two classes of uncertain singular time-delay systems," IEEE Transactions on Automatic Control, vol. 52, no. 5, pp. 880-885, 2007.

[7] S. L. Campbell and V. H. Linh, "Stability criteria for differential-algebraic equations with multiple delays and their numerical solutions," Applied Mathematics and Computation, vol. 208, no. 2, pp. 397415, 2009.

[8] E. Fridman, "Stability of linear descriptor systems with delay: a Lyapunov-based approach," Journal of Mathematical Analysis and Applications, vol. 273, no. 1, pp. 24-44, 2002.

[9] A. Haidar and E. K. Boukas, "Exponential stability of singular systems with multiple time-varying delays," Automatica, vol. 45, no. 2, pp. 539-545, 2009.

[10] Z. Wu and W. Zhou, "Delay-dependent robust $H_{\infty}$ control for uncertain singular time-delay systems," IET Control Theory E Applications, vol. 1, no. 5, pp. 1234-1241, 2007.

[11] S. Xu, P. van Dooren, R. Ştefan, and J. Lam, "Robust stability and stabilization for singular systems with state delay and parameter uncertainty," IEEE Transactions on Automatic Control, vol. 47, no. 7, pp. 1122-1128, 2002.

[12] D. Yue and Q. L. Han, “Delay-dependent robust $H_{\infty}$ controller design for uncertain descriptor systems with time-varying discrete and distributed delays," IEE Control Theory and Applications, vol. 152, no. 6, pp. 628-638, 2005.

[13] S. Zhu, Z. Li, and C. Zhang, "Delay decomposition approach to delay-dependent stability for singular time-delay systems," IET Control Theory E Applications, vol. 4, no. 11, pp. 2613-2620, 2010.

[14] M. C. de Oliveira, J. Bernussou, and J. C. Geromel, "A new discrete-time robust stability condition," Systems \& Control Letters, vol. 37, no. 4, pp. 261-265, 1999. 


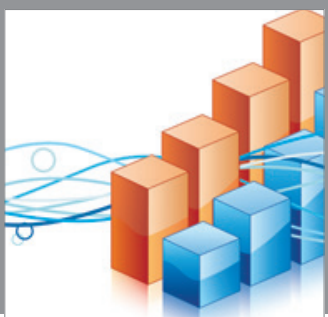

Advances in

Operations Research

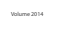

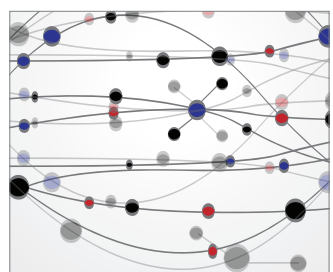

\section{The Scientific} World Journal
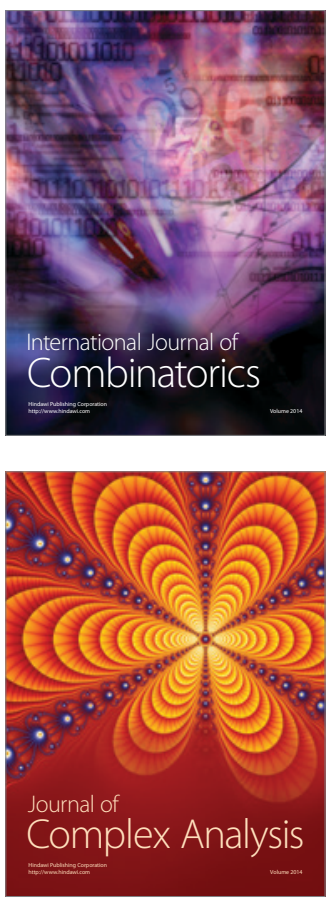

International Journal of

Mathematics and

Mathematical

Sciences
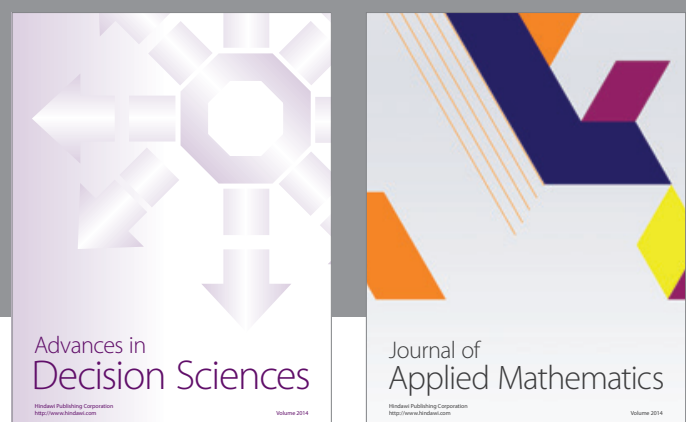

Journal of

Applied Mathematics
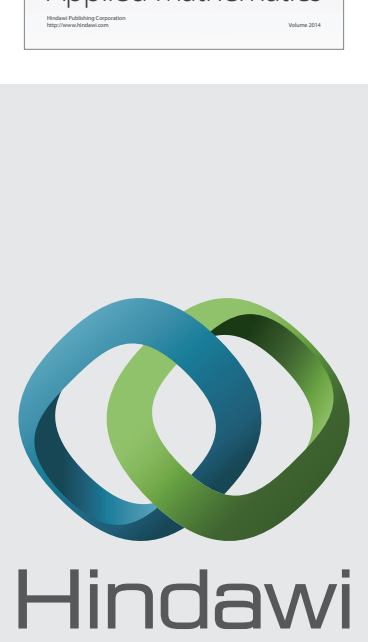

Submit your manuscripts at http://www.hindawi.com
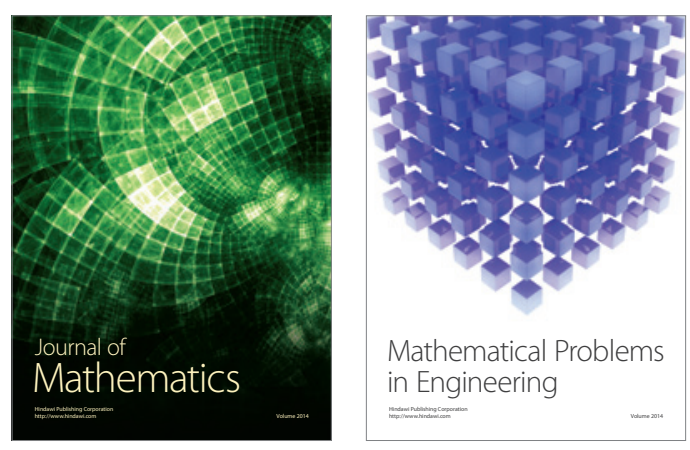

Mathematical Problems in Engineering
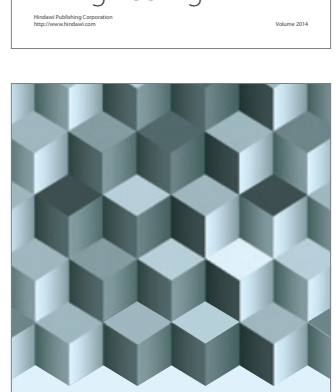

Journal of

Function Spaces
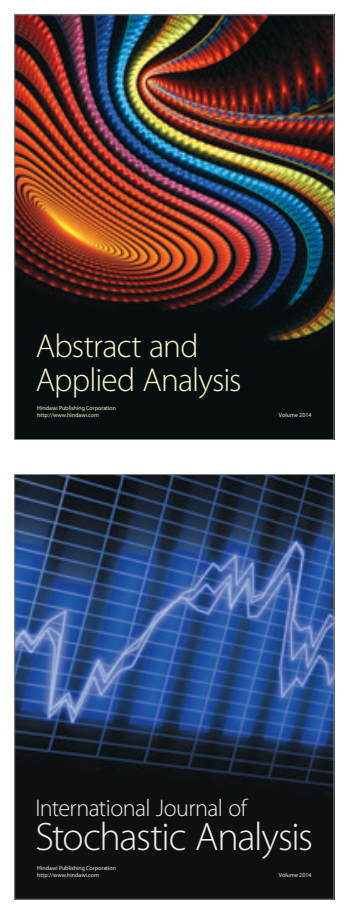

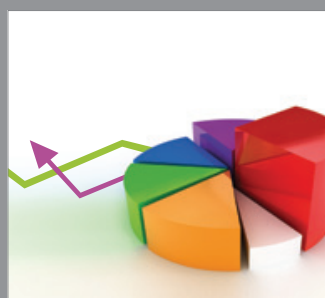

ournal of

Probability and Statistics

Promensencen
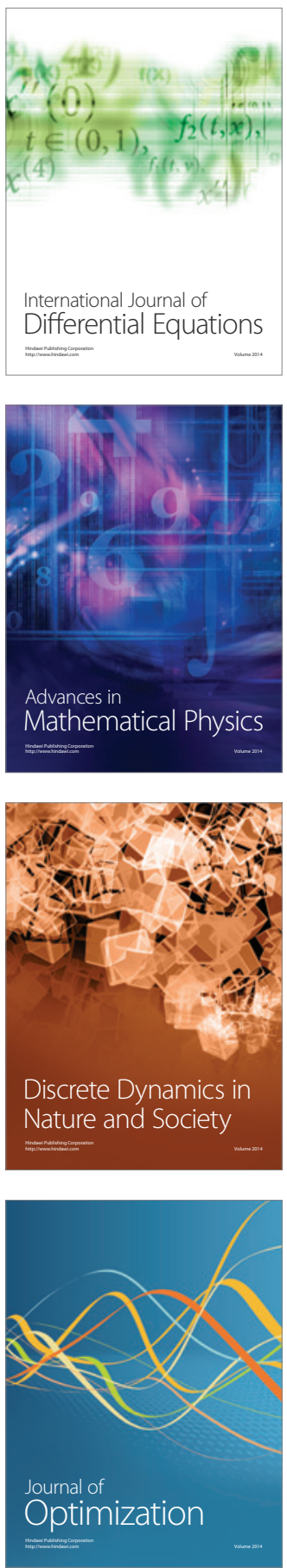